\title{
Effect of A Vinyl Ester-Carbon Nanotubes Sizing Agent on Interfacial Properties of Carbon Fibers Reinforced Unsaturated Polyester Composites
}

\author{
Zijian $\mathrm{Wu}^{1,2}$, Lin $\mathrm{Li}^{1}$, Ning Guo ${ }^{2 *}$, Rongchen Yang ${ }^{1}$, Dawei Jiang ${ }^{4}$, Mingyu Zhang ${ }^{1}$, Mingyan Zhang ${ }^{1,2}$, Yudong Huang ${ }^{3}$ and Zhanhu Guo ${ }^{5 *}$
}

A vinyl-functionalized carbon nanotubes (CNTs)-containing vinyl ester sizing agent was prepared for improving the interlaminar shear strength (ILSS) and impact properties of carbon fibers (CFs) reinforced unsaturated polyester (UP) composites. More reactive unsaturated double bonds and better interfacial compatibility make vinyl ester R806 a more suitable resin for preparing sizing agent for CF/UP composites when compared to the commercial epoxy sizing agent. The surface characteristics of CFs and the interfacial properties of the composites before and after sizing agent treatment were investigated. The observed uniformly dispersed CNTs-containing sizing agent on the CFs surface obviously increased the surface roughness. The amount of polar functional groups and the wettability of CFs were significantly enhanced after the coating treatment. The ILSS and impact toughness were enhanced by 28.4 and $53.3 \%$, respectively. The sizing agent effectively enhanced the interfacial adhesion by improving the surface energy, and increasing chemical bonding and mechanical interlocking.

Keywords: Carbon fibers; Coating; Interphase; Mechanical properties

Received 11 October 2019, Accepted 28 November 2019

DOI: $10.30919 / \mathrm{esmm} 5 \mathrm{f} 601$

\section{Introduction}

Advances in surface appearance, production efficiencies and overall part quality were required to keep thermoset composites competitive as key materials for the automotive industry. In response to these challenges, a superior molding resin has been developed to meet the demanding variety of requirements. unsaturated polyester (UP) MR13006 from Ashland company lends itself well to the production of SMC, TMC and BMC moldings.

Owing to the radical polymerization, UP has a better curing efficiency than common epoxy resin. ${ }^{1,2}$ Some UP can finish curing within $1 \mathrm{~min}$ and MR13006 from Ashland is just this kind of UP. In order to have a higher production rate per each used mold, the curing efficiency becomes more important for manufacturing enterprises. ${ }^{3}$ Meanwhile, the mechanical properties of $\mathrm{CF} / \mathrm{UP}$ composites are preferred to be close to the level of CF/epoxy composites. The ultimate performance of composites is largely dependent on the interfacial

College of Material Science and Engineering, Harbin University of Science and Technology, Harbin, 150040, China

Key Laboratory of Engineering Dielectrics and Its Application, Ministry of Education, Harbin University of Science and Technology, Harbin, 150040, China

School of Chemical Engineering and Technology, Harbin Institute of Technology, Harbin 150001, China

${ }^{4}$ Heilongjiang Key Laboratory of Molecular Design and Preparation of Flame Retarded Materials, Northeast Forestry University, Harbin 150040, China

Integrated Composites Lab (ICL), Department of Chemical and Biomolecular Engineering, University of Tennessee, Knoxville, TN, 37996, USA

*E-mail:tad@hrbust.edu.cn; zguo10@utk.edu properties. ${ }^{4,5}$ Nevertheless, the relatively weak interlaminar adhesion between CFs and UP was observed to result in a low ILSS and impact toughness. ${ }^{6,7}$

Polymer nanocomposites are promising in many fields from the viewpoint of their inherent excellent properties. ${ }^{6-20}$ In the field of $\mathrm{CF}$ composites, the interphase containing nanomaterials (e.g., CNTs or Graphene) is helpful to transfer stress and creates mechanical interlocking between $\mathrm{CF}$ and resin. ${ }^{21}$ Various kinds of surface treatment approaches have been employed to introduce nanomaterials into the interface of composites, such as chemical grafting, ${ }^{22-24}$ coating with sizing agent, ${ }^{25}$ chemical vapor deposition (CVD), ${ }^{26}$ electrochemical deposition(ECD) ${ }^{11}$ and electrophoretic deposition (EPD). ${ }^{11,26} \mathrm{CVD}$ is the only method that can be used to grow carbon nanofillers on carbon fiber surface, but the eutectic melting between metal catalyst and carbon leads to a huge decrease of tensile strength of single fiber. EPD is a simple technique for deposition of carbon nanofillers on fiber surface based on electrostatic adsorption mechanism. However, the relatively weak interaction between carbon nanofillers and fibers is not benecicail to improve interfacial adhesion. Compared to other approaches, sizing agent approach can be based on a classical commercial sizing agent, or on a polymer matrix that will be deposited as a thin layer over the fiber surface, and introducing no damage to the fibers. ${ }^{27}$ For an enhanced interfacial compatibility, the first priority for preparing a sizing agent is to select a suitable main polymer. ${ }^{28}$ Epoxy is widely applied to prepare commercial sizing agent for most $\mathrm{CF}$ enterprises, which is due to the dominant position of epoxy in the field of matrix resin. Epoxy sizing agent has proven to be an effective method to enhance the interfacial properties of CF/epoxy composites. But for UP MR13006, the interfacial compatibility between epoxy sizing agent and matrix has been studied less extensively. It remains to be seen whether the better polymer than epoxy could be found to prepare sizing agent for $\mathrm{CF} / \mathrm{UP}$ composites. In this paper, three kinds of polymers were used to prepare 
sizing agents according to either their wide applications for sizing agent preparation or similar chemical structure with UP, including widely used epoxy, UP (MR13006), and a vinyl ester (R806) to disclose the effects of polymer structure on the interfacial properties of $\mathrm{CF} / \mathrm{UP}$ composites.

A comparison study indicated that R806 was the most effective for interfacial property enhancement. The massive hydroxyl and vinyl groups and relative low viscosity $(<0.23 \mathrm{~Pa}$.s $)$ of R806 warrant a good spreadability on the fiber surface. The unsaturated double bonds of R806 make it easier to participate in the curing reaction with UP matrix. $\mathrm{CNT}$ is considered to be a promising reinforcing material because of its

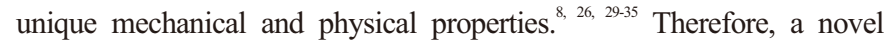
sizing agent containing vinyl functionalized CNTs and vinyl resin R806 was prepared for enhancing the interface performance of CF/UP. The effects of this novel sizing agent on surface characteristics of CFs were studied by XPS, FTIR and SEM, and the interfacial adhesion of final $\mathrm{CF} / \mathrm{UP}$ was discussed with ILSS and impact test.

\section{Experimental}

\subsection{Materials}

Un-sizing CFs (raw CFs) were obtained from Guangwei Co., China (12 $\mathrm{K}$, diameter: $7 \mu \mathrm{m}$ ). CNTs (diameter: $8-15 \mathrm{~nm}$ and length: 0.5-2 $\mu \mathrm{m}$ ) were obtained from Chinese Academy of Sciences, Chengdu. MR13006 (Volume shrinkage: 8.5\%) and low shrinkage agent LP4016 (vinyl acetate polymer) were obtained from Ashland China. Vinyl ester R806 (Volume shrinkage: 7.5\%) was purchased from SHOWA HIPOLYMER Co., China. Water-soluble $\mathrm{EP}^{36}$ (volume shrinkage: $\%$, solid content $50 \%$ ) was prepared in our lab and the corresponding amine hardendner were supplied by Guangwei Co., China. Tert-butyl peroxybenzoate (TBPB) from ACROS ORGANICS was used as an initiator for the CF/UP system. N-(3-Dimethylaminopropyl)-N'-ethylcarbodiimide (EDC), $\mathrm{SOCl}_{2}$, acetone, acryloyl chloride, acrylamide, DMF, THF, $\mathrm{H}_{2} \mathrm{SO}_{4}(98 \%), \mathrm{HNO}_{3}(68 \%)$ and ammonium bicarbonate were purchased from Changzheng Co., China. Figs. $3 \mathrm{a}-\mathrm{c}$ shows the molecular formulas of three kinds of main polymers for preparing sizing agents.
2.2 Preparation of sizing agents containing different main polymers 2.2.1 Sizing agents containing water-soluble EP

$4 \mathrm{~g}$ water-soluble EP, and amine curing agent ( $1 \mathrm{wt} \%$ water-soluble EP) were dispersed in deionized water in a sonication bath for $15 \mathrm{~min}$ to prepare $200 \mathrm{~g}$ sizing agent, the content of main polymer in sizing agent was $1 \mathrm{wt} \%$.

\subsubsection{Sizing agents containing MR13006}

$10.5 \mathrm{~g}$ MR13006, $4.5 \mathrm{~g}$ LP4016, and $0.15 \mathrm{~g}$ TBPB were mixed uniformly and $2 \mathrm{~g}$ such mixture was dispersed in acetone in a sonication bath for $15 \mathrm{~min}$ to prepare $200 \mathrm{~g}$ sizing agent, the content of main polymer in sizing agent was $1 \mathrm{wt} \%$ as well.

\subsubsection{Sizing agents containing $R 806$}

$15 \mathrm{~g}$ R806 and $0.3 \mathrm{~g}$ TBPB were mixed uniformly and $2 \mathrm{~g}$ such mixture was dispersed in acetone in a sonication bath for $15 \mathrm{~min}$ to prepare 200 $\mathrm{g}$ sizing agent, the content of main polymer in sizing agent was $1 \mathrm{wt} \%$.

\subsection{Preparation of CNTs-Vinyl}

The CNTs used in this study were dispersed and oxidized in the mixed acids $\left(\mathrm{V}_{(\mathrm{HNO} 3)}: \mathrm{V}_{(\mathrm{H} 2 \mathrm{SO} 4)}=6: 5\right)$ at $65{ }^{\circ} \mathrm{C}$ for $8 \mathrm{~h}$. After treatment, the CNTs were filtered, washed with deionized water to reach $\mathrm{pH} 7$ and dried at $60 \mathrm{C}$ for $8 \mathrm{~h}$ to get carboxyl functionalized CNTs (CNTs-COOH). After freeze drying treatment, $2 \mathrm{~g}$ CNTs- $\mathrm{COOH}$ were added to $60 \mathrm{ml}$ lithium aluminum hydride-diethyl ether saturated solution under reflux conditions for $24 \mathrm{~h}$. After reaction, the CNTs were filtered, washed with deionized water and freeze-dried to get hydroxyl functionalized CNTs $(\mathrm{CNTs}-\mathrm{OH}), \mathrm{CNTs}-\mathrm{OH}$ further react with acryloyl chloride to get vinyl functionalized CNTs (CNTs-Vinyl).

\subsection{Preparation of CNTs-Acryl}

CNTs-COOH, acrylic amide, EDC and THF were mixed uniformly at room temperature for $48 \mathrm{~h}$ to get Acryl functionalized CNTs (CNTs-Acryl). Mixing the acrylic amide, EDC and CNTs in the weight ratio of 1: 1: 2.

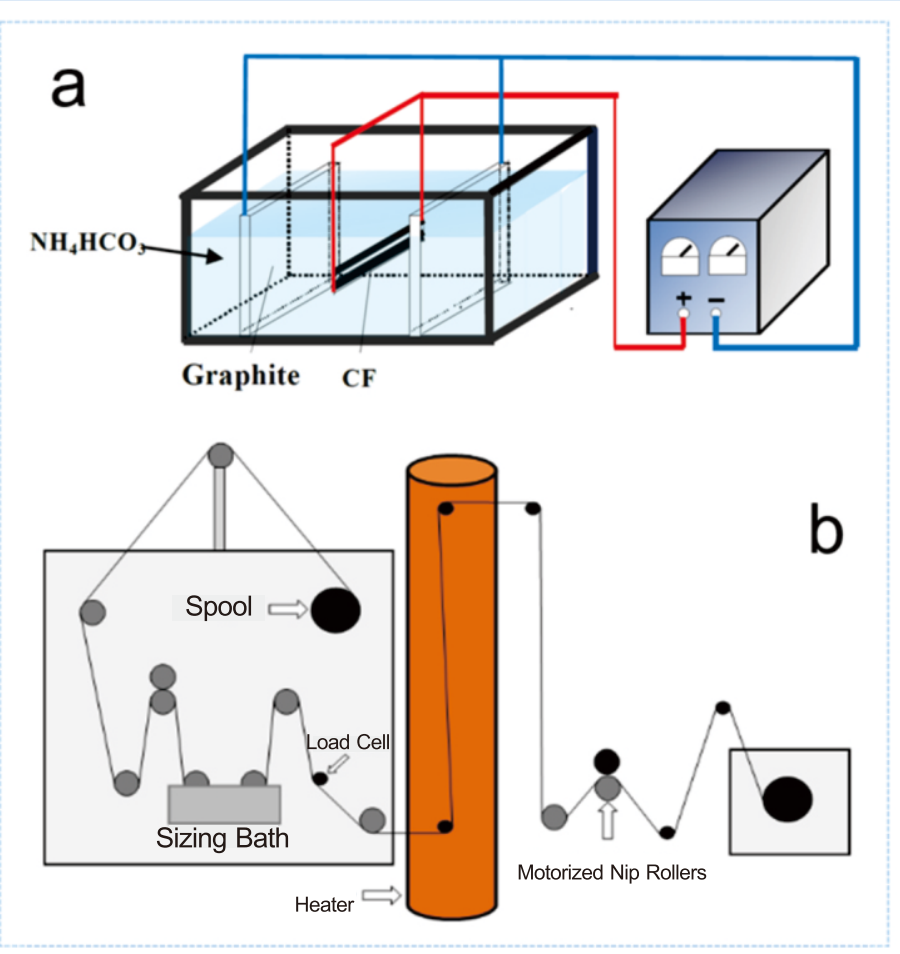

Fig. 1 The sketch of (a) the self-made anodic oxidation equipment and (b) the lab scale sizing equipment. 


\subsection{Preparation of sizing agents containing CNTs-Acryl and R806}

$1 \mathrm{~g}$ CNTs-Acryl and $2 \mathrm{~g}$ R806 mixture were dispersed in acetone in a sonication bath for $15 \mathrm{~min}$ to prepare $200 \mathrm{~g}$ sizing agent (R806-CNTsAcryl), the content of main polymer R806 in sizing agent was $1 \mathrm{wt} \%$ and CNTs-Acryl content was $0.5 \mathrm{wt} \%$.

\subsection{Sizing treatment and the preparation of composites}

The CFs were treated with a self-made anodic oxidation equipment (Fig. 1a) for $4 \mathrm{~min}$ in 5\% ammonium bicarbonate solution with an electric current density of $300 \mathrm{~mA} / \mathrm{g}$. After being washed and dried, the CFs were coated with R806-CNTs-Acryl with a lab scale sizing equipment (Fia. 1b).

The resin system was prepared by mixing MR13006, low shrinkage agent LP4016 and initiator TBPB in a ratio of 105:45:1. The prepreg containing the surface modified $\mathrm{CFs}$ and the resin system was transferred into a mould for curing process. Such prepreg was maintained at $80{ }^{\circ} \mathrm{C}$ for half an hour without pressurizing and then $140{ }^{\circ} \mathrm{C}$ for $1 \mathrm{~h}$ under $10 \mathrm{MPa}$. The resin content of the final product should be restricted to $35 \pm 1 \mathrm{wt} \%$ as suggested for Ashland company.

\subsection{Characterizations}

The fiber surface morphology was observed with SEM (FE-SEM, JEOL JSM-6335F, Japan). XPS (Thermo Scientific, USA) was employed to investigate the surface chemical composition of CNTs and CFs using a monochromated $\mathrm{Al} \mathrm{Ka}$ source $(1486.6 \mathrm{eV})$ at a base pressure of $2 \times 10^{-9}$ mbar. Dynamic contact angle (DCA) was measured by a CA meter (DCAT21, Data-Physics Instrument, Germany). Deionized water $\left(\gamma^{\mathrm{d}}\right.$ $\left.=21.8 \mathrm{~mJ} / \mathrm{m}^{2}, \gamma=72.8 \mathrm{~mJ} / \mathrm{m}^{2}\right)$ and diiodomethane $\left(\gamma^{\mathrm{d}}=50.8 \mathrm{~mJ} / \mathrm{m}^{2}, \gamma=50.8\right.$ $\mathrm{mJ} / \mathrm{m}^{2}$ ) were employed as test medium. CA was calculated by mass variation when $\mathrm{CF}$ was immersed to the test medium based on Wilhelmy's Equation 2, the sketch of the testing process is illustrated in Fig. 2.

Based on ASTM D2344, the ILSS tests were carried out on a mechanical testing machine (5569, Instron, USA) at a cross-head speed of $2 \mathrm{~mm} / \mathrm{min}$. The specimen dimensions for ILSS test were $20 \mathrm{~mm} \times$ $6.5 \mathrm{~mm} \times 2 \mathrm{~mm}$. An impact test system (9250HV, Instron, USA) was used to characterize the impact toughness of composites with impact span of $40 \mathrm{~mm}$. The specimen dimensions for impact test were $55 \mathrm{~mm}$ $\times 6.5 \mathrm{~mm} \times 2 \mathrm{~mm}$. The drop velocity and drop weight were of $1 \mathrm{~ms}^{-1}$ and $3 \mathrm{~kg}$, respectively. The recorded value of ILSS and impact toughness for each group of specimens was averaged from the data of 5 successful measurements.

\section{Results and discussion}

\subsection{Surface topography and chemical composition of CFs}

Figs. 3(a-c) shows the topography and oxygen element linear distribution of CFs. The sizing agents containing R806 and watersoluble EP exhibited good spreadability on the CF surface. Compared to other samples, the interfacial compatibility of CFs with the sizing agent containing MR13006 is worse. As shown in Fig. 3a, the distribution curve of oxygen element had a larger fluctuation, showing that the MR13006 sizing agent cannot be distributed uniformly on the CF surface.

Figs. 3f-h shows the C1s XPS spectra of the CFs coated with different sizing agents. The $\mathrm{C} 1 \mathrm{~s}$ spectrum of the $\mathrm{CFs}$ coated with watersoluble EP demonstrated $57.4 \% \mathrm{C}-\mathrm{C}, 41.9 \% \mathrm{C}-\mathrm{O}$ and $0.7 \% \mathrm{O}-\mathrm{C}=\mathrm{O}$ bonds, the $\mathrm{C}-\mathrm{O}$ content is much higher than that of $\mathrm{O}-\mathrm{C}=\mathrm{O}$ bond. Similarly, the $30.5 \%$ C-O content on the CFs coated with R806 is also relatively high, In contrast, the $\mathrm{C} 1 \mathrm{~s}$ spectrum of the $\mathrm{CFs}$ coated with MR13006 consisted of $75.5 \% \mathrm{C}-\mathrm{C}, 13.9 \% \mathrm{C}-\mathrm{O}$ and $10.6 \% \mathrm{O}-\mathrm{C}=\mathrm{O}$ bonds, the content of $\mathrm{O}-\mathrm{C}=\mathrm{O}$ bond is the highest among all the samples, and the $\mathrm{C}-\mathrm{O}$ bonds are relatively less. As shown in Figs. 3(ac), each repetitive structure unit of the epoxy molecular includes one hydroxyl group, and two end epoxy groups. No $\mathrm{O}-\mathrm{C}=\mathrm{O}$ bonds have been found in the molecular structure. Vinyl ester is the reaction product of epoxy and unsaturated monoacids, thus R806 have remarkable structure features of the epoxy, possessing relatively high quantity of C$\mathrm{O}$ bonds.

SEM images (Figs. 3a-c) reveals that the epoxy sizing agent has the best uniformity on the fiber surface, which is attributed to plenty of hydroxyl groups of epoxy polymer chains. It should be noted that there is no ester group in epoxy molecular structure, confirming that hydroxyl groups have a better interfacial compatibility with fiber surface treated with anodic oxidation than ester groups. This is the reason for choosing epoxy to prepare the sizing agent in most commercial carbon fiber production enterprises. R806 keep the structural features of epoxy backbone, which are characterized by multiple hydroxyl groups.

Therefore, compare to MR13006, R806 have better spreading property on the fiber surface (Figs. 3a-c). Note that MR13006 with the highest oxygen content shows the lowest $\mathrm{C}-\mathrm{O}$ content and the $\mathrm{O}-\mathrm{C}=\mathrm{O}$ content of MR13006 is the highest among three kinds of main polymers. As shown in Fig. 3a, the MR13006 is hard to be distributed
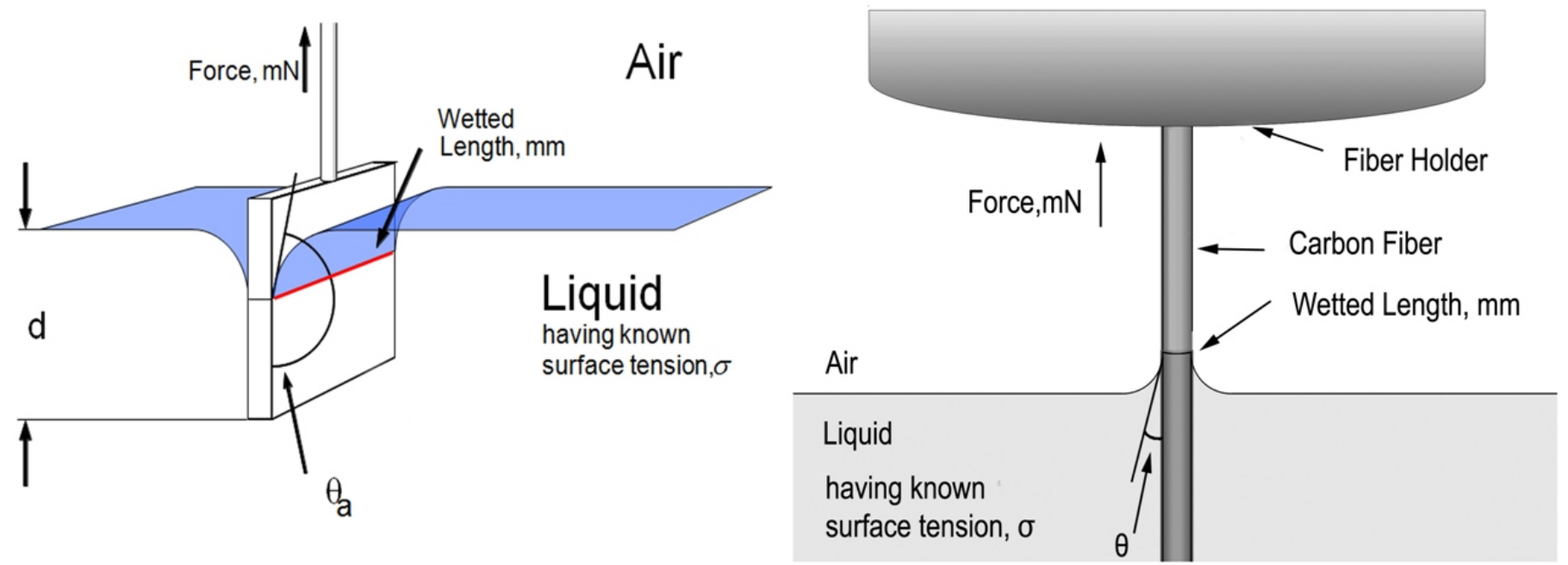

Fig. 2 The sketch of the DCA testing process. 
uniformly on the fiber surface, indicating that the $\mathrm{C}-\mathrm{O}$ groups have a better compatibility for carbon fiber surface than the $\mathrm{O}-\mathrm{C}=\mathrm{O}$ groups. From the structure schematic of MR13006 (Fig. 3a), it can be seen that the hydroxyl groups appear only at the ends of molecular chain and the repeating units of molecular chain contain a large amount of ester groups and carbonyl groups, which agree well with the fitted curves of XPS C1s. Moreover, the length of the molecular chain for different polymer is one important factor to influence the surface coating. R806 and the epoxy used in this paper have a similar number average molecular mass $(<1000 \mathrm{~g} / \mathrm{mol})$. However, the molecular mass of MR13006 is about $3000 \mathrm{~g} / \mathrm{mol}$, which means that a longer molecular chain cannot get inside the tiny grooves and voids.

\subsection{ILSS and impact toughness of CF/UP composites}

Figs. 1d\&e shows the ILSS and impact toughness of CF/UP composites before and after coating. As shown in Fig. 1d, the load is approximately linear to the displacement in the initial period of loading. With further increasing the displacement, the load declines sharply from peak value, which is probably due to the slipping between the fibers and the UP resin or the yielding of UP. Anodic oxidation is an effective means to increase the roughness and reactive activity of fiber surface, which is helpful to enhance the interface adhesion and interlocking between $\mathrm{CF}$ and UP. It was discovered that the fiber after anodic oxidation could make ILSS increase to $50.3 \mathrm{MPa}$ from the original $47.7 \mathrm{MPa}$. With the benefit of massive hydroxyl groups, the sizing agent containing R806 and epoxy can distribute uniformly on the CF surface. Based on previous analysis, the sizing agent containing MR13006 has the lowest hydroxyl group content, which is hard to obtain a good wettability on the fiber surface. Moreover, the molecular weight and viscosity of MR13006 are much higher than those of other three main polymers for preparing sizing agent, which is an obstacle for short range contact between the molecular chain and the fiber surface. The interface layer with the gradient variation of mechanical properties is beneficial to transfer and disperse stress. However, the exorbitant molecular weight is not helpful to form such gradient interface layer ${ }^{37}$. The matrix resin is composed of MR13006 (molecular weight: $3000 \mathrm{~g} / \mathrm{mol}$ ) and LP4016 (molecular weight: $40000 \mathrm{~g} / \mathrm{mol}$ ). The exorbitant molecular weight of resin mixture will limit the movement of polymer molecular chains from the interface region to the matrix resin region. The ILSS of CF/UP composites treated with MR13006 sizing agent is just 52.3 MPa. The relatively low molecular weight of R806 makes them more easily form the interface layer with the gradient mechanical properties. Owing to
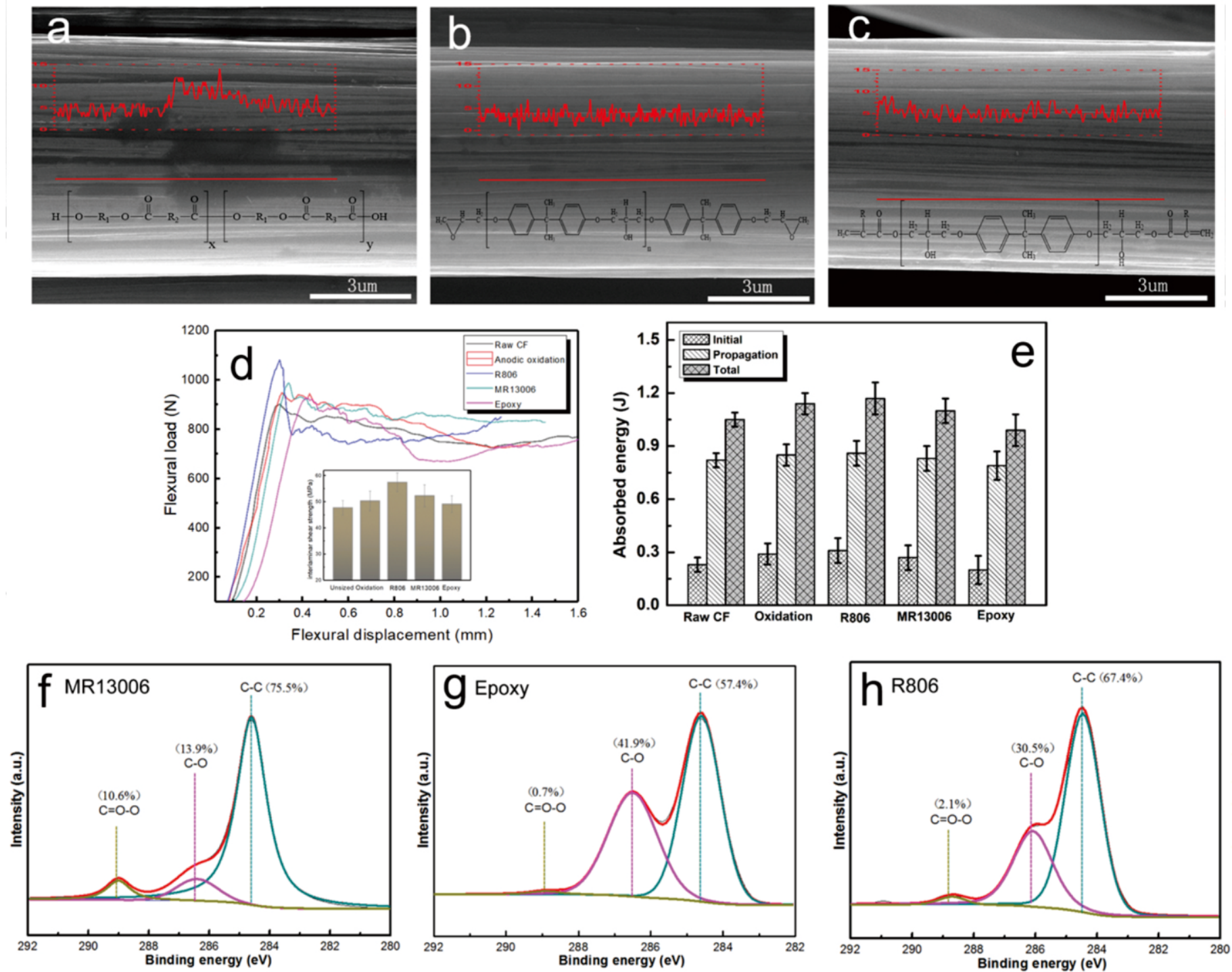

Fig. 3 SEM images of (a) CF coated with MR13006, (b) CF coated with epoxy, (c) CF coated with R806, (d) ILSS and (e) the impact toughness of CF/UP composites, C1s spectra of XPS: (f) CF coated with MR13006, (g) CF coated with epoxy, (h) CF coated with R806. 
good wettability, the ILSS of R806 sizing agent was $57.4 \mathrm{MPa}$. The good interface bonding stems from the molecular structure and physicochemical property of polymer themselves. A large amount of hydroxyl groups and relatively low molecular weight of R806 assure the good wettability of sizing agent on the fiber surface. Unsaturated double bonds coexist in vinyl resin, UP resin and styrene solvent, and similar chemical structure is beneficial to enhance the interface compatibility. More importantly, unsaturated bonds can participate in the curing process of UP matrix through the reaction of radical polymerization, and generate solid interphase linked with covalent bonds. However, the impact toughness of CF/UP composite has not been improved much by R806 sizing treatment.

It is worth noting that the epoxy sizing agent with the lowest volume shrinkage has the worst ILSS. Epoxy is widely applied to prepare commercial sizing agent for most CF enterprises. Epoxy sizing agent can effectively improve the interface compatibility for the fibers reinforced composites, especially for the carbon fibers reinforced epoxy systems. But obviously, vinyl resin sizing agents have a better interface compatibility for the $\mathrm{CF} / \mathrm{UP}$ composites.

\subsection{The design of sizing agents containing CNTs-Acryl and R806}

R806 is chosen as the main polymer to prepare sizing agent containing CNTs-Acryl for the CF/UP composites. Introducing nanoparticles to the interface of $\mathrm{CF}$ reinforced polymer composites has been proved to be an effective way to toughen and reinforce composites. Fig. 4 shows the chemical reaction diagram for R806-CNTs-Acryl in the CF/UP composites. Firstly, CFs were treated with anodic oxidation, and then the unsaturated bonds were chemical grafted to the fiber surface through reacting with active groups caused by electrochemical oxidation. The final carbon fibers functionalized with unsaturated bonds were abbreviated as CF-Vinyl. The unsaturated bonds were introduced to the surface of CNTs through the similar process as mentioned above, abbreviated as CNTs-Acryl. In this way, both the double bonds on CFVinyl surface and the double bonds on CNTs-Acryl surface could be involved into the curing reaction of MR13006 through free radical polymerization process and could create chemical bonding in different gradations in the interface area. In order to shorten the curing time, high temperature curing agent TBPB was chosen for MR13006 curing at $140{ }^{\circ} \mathrm{C}$ as recommended by the Ashland Company. High temperature solidification is helpful to promote the interactions or to create chemical bonding between polar groups. R806 molecular chain acts as a bridge between $\mathrm{CF}$ and MR13006, whose one end is connected to the fiber surface by chemical bonds and the other end is involved in the curing process by unsaturated double bonds.

\subsection{The unsaturated bond-functionalization CNTs}

The schematic of the surface functionalization process of CNTs-Vinyl and CNTs-Acryl are shown in Fig. 5, respectively. The corresponding experiment procedure is described in experimental part. Figs. $6 \mathrm{a} \& \mathrm{~b}$ shows the SEM images of CNTs-Vinyl and CNTs-Acryl, respectively.
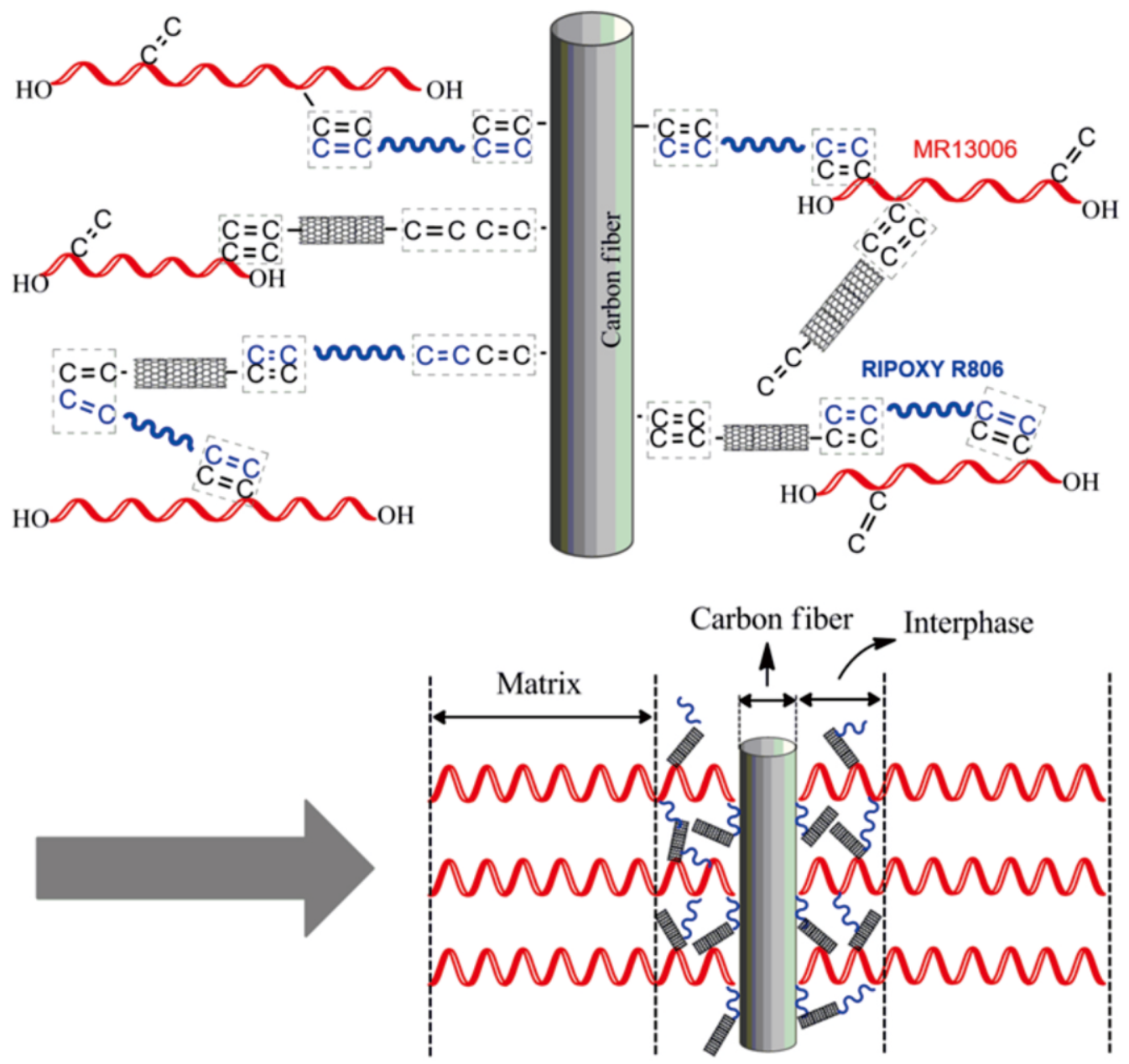

Fig. 4 The chemical reaction diagram for R806-CNTs-Acryl in the CF/UP composites. 
As shown in Fig. 6, the CNTs-Vinyl cannot be dispersed in polar solvent uniformly when compared to CNTs-Acryl and generated obvious agglomeration. In sharp contrast with CNTs-Vinyl, the CNTsAcryl can disperse very well in polar solvent. The surface morphology and dispersibility of CNTs-Acryl are similar with CNTs-COOH. On the one hand, more reaction procedure means more dispersion and drying treatments for chemical grafting process, which increase the aggregation risk for CNTs. On the other hand, owing to abundant amido bonds created by surface grafting process, the surface polarity of CNTs is improved, which is beneficial to increase the compatibility of CNTs to the polar solvent or polymer matrix.

As shown in Figs. $7 \& 8$, XPS was employed to illustrate the change of surface functional groups of CNTs before and after the unsaturated bond-functionalization. Compared to Raw CNTs, an obvious O1s peak, which belongs to CNTs-COOH, could be observed after the oxidation treatment. An obvious N1s peak was observed around $400 \mathrm{eV}$, which confirm the reaction between acrylic amide and carboxylic group.

Chemical grafting could be further assessed by $\mathrm{C} 1 \mathrm{~s}$ spectra, as shown in Figs. 8a, b\&c. For raw CNTs, the characteristic peak at 284.6, 285.3 and $290.4 \mathrm{eV}$ corresponds to the $\mathrm{C}=\mathrm{C}, \mathrm{C}-\mathrm{C}$ and $\pi-\pi^{*}$, respectively, all of which belong to the characteristic peak of graphite. After oxidation treatment, a large amount of oxygen-containing functional groups could be observed, such as $\mathrm{C}-\mathrm{OH}$ at $286.5 \mathrm{eV}$ and $\mathrm{O}-$ $\mathrm{C}=\mathrm{O}$ at $288.8 \mathrm{eV}$. For CNTs-Acryl, the new $\mathrm{C} 1$ s peak at $287.2 \mathrm{eV}$ was assigned to the $\mathrm{N}-\mathrm{C}=\mathrm{O}$, indicating that acrylamide was covalently linked with CNTs surface by the chemical bonds. Based on the above the results and discussions, CNTs-Acryl were chosen for the preparation of the sizing agent.

\subsection{The effect of R806-CNTs-Acryl on surface properties of CFs} Figs. 9a\&b shows the surface morphology of CFs coated with R806 sizing agent containing raw CNTs and CNTs- Acryl, respectively. CNTs- Acryl has a better dispersion in sizing agent than raw CNTs and no obvious aggregations were observed in Fig. 9b. The surface roughness (Ra) of the fibers treated with R806-CNTs-Acryl was obviously increased in comparison with those treated with R806 sizing agent containing raw CNTs. The variation of surface polar groups influences the surface free energy and its components. The higher surface energy means that the matrix can be uniformly dispersed on the fiber surface, which is beneficial to enhancing the interfacial compatibility of composites.
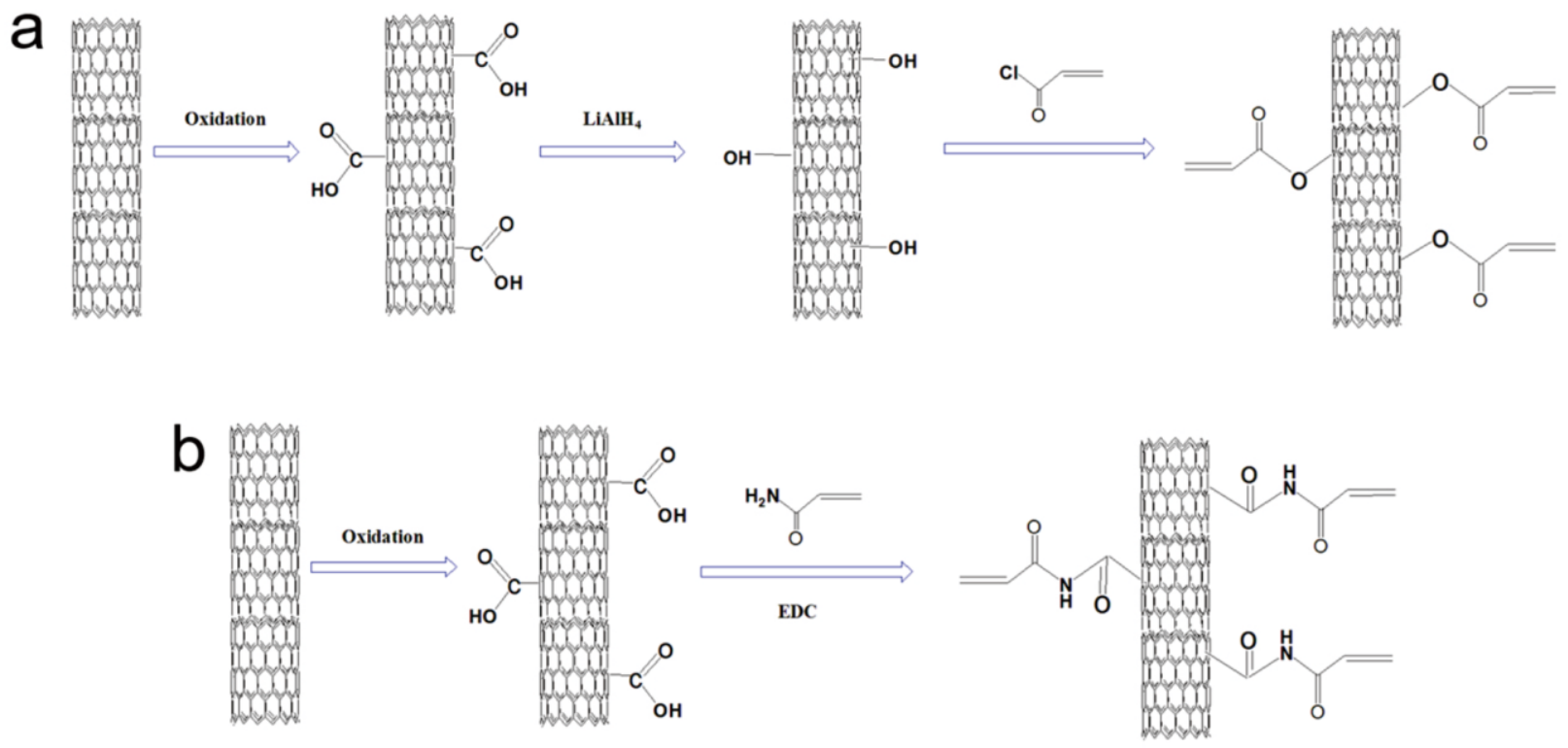

Fig. 5 Schematic of surface functionalization process of CNTs: a) CNTs-Vinyl, b) CNTs-Acryl.
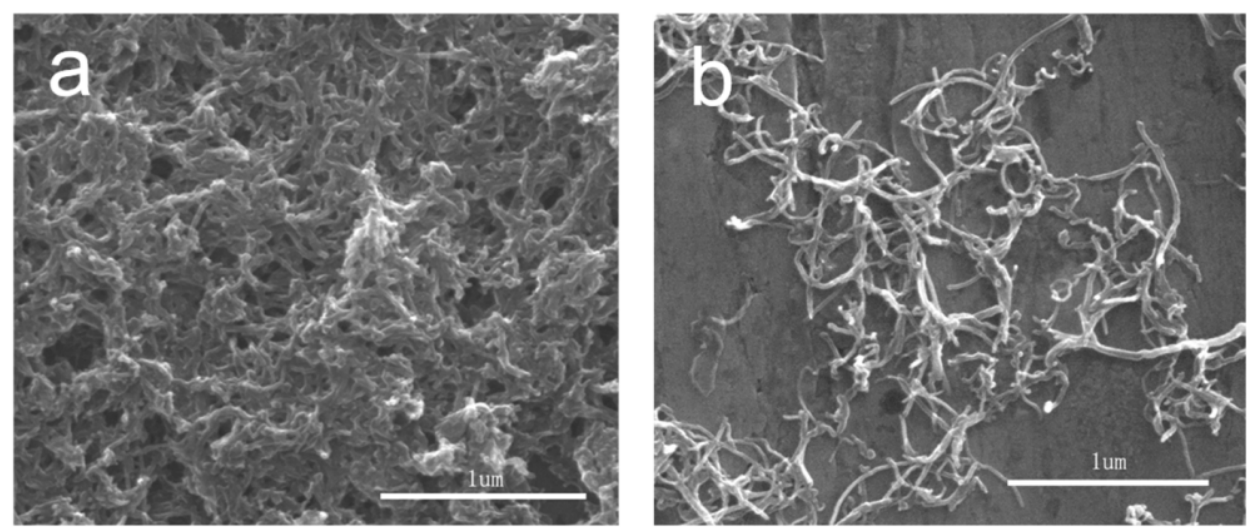

Fig. 6 SEM images of CNTs: a) CNTs-Vinyl, b) CNTs-Acryl. 


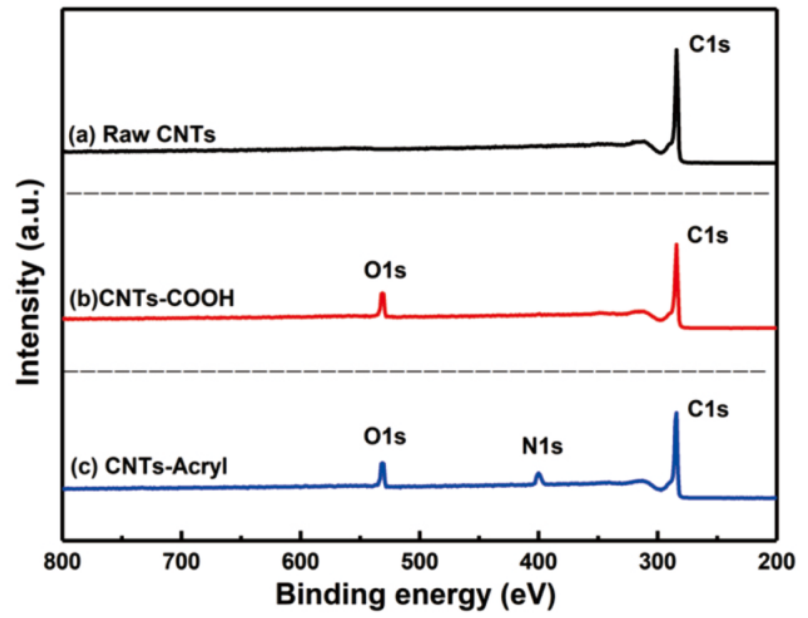

Fig. 7 XPS wide scans of CNTs.
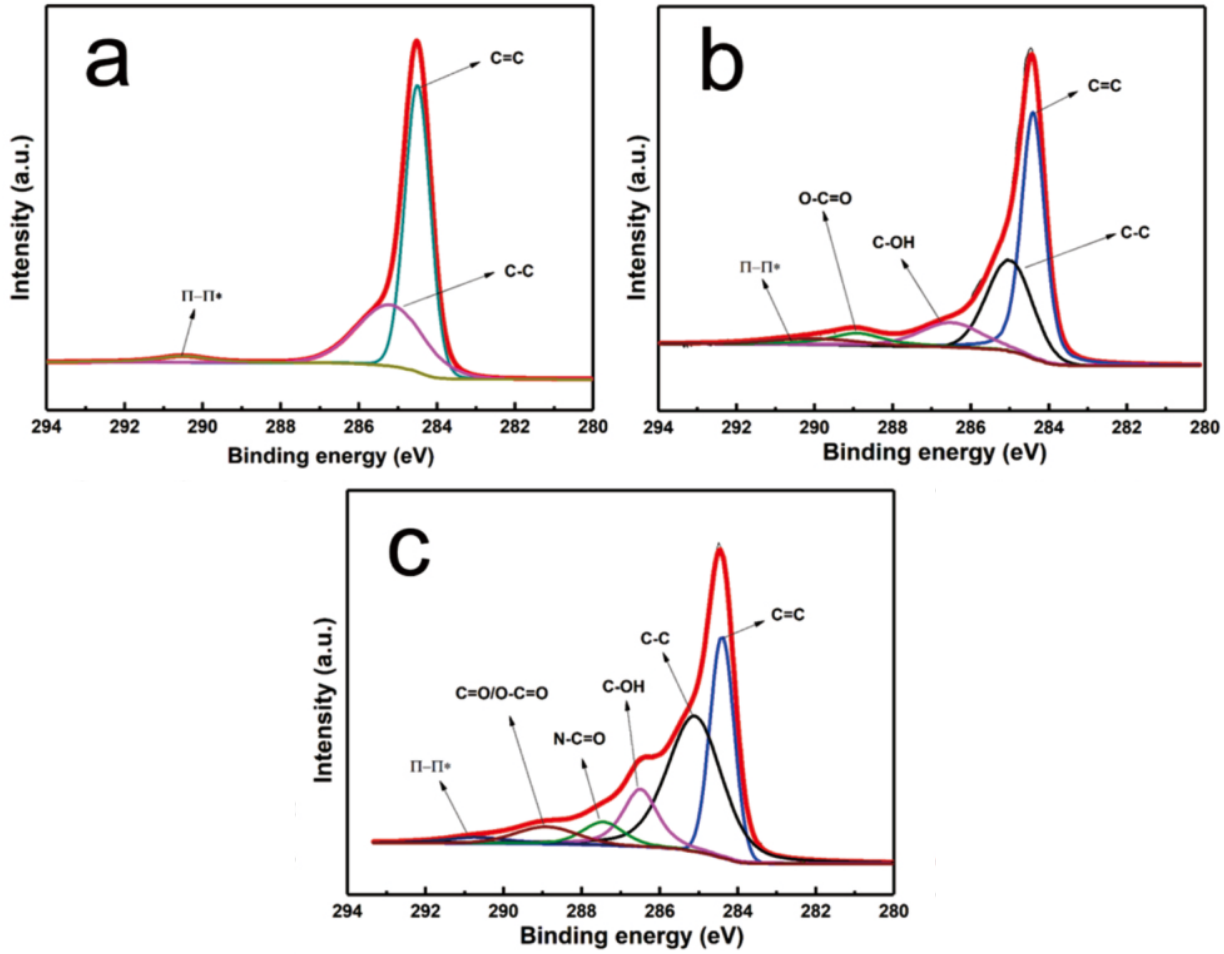

Fig. 8 Fitting curves of C1s spectra of CNTs: a) Raw-CNTs, b) CNTs-COOH, c) CNTs-Acryl.
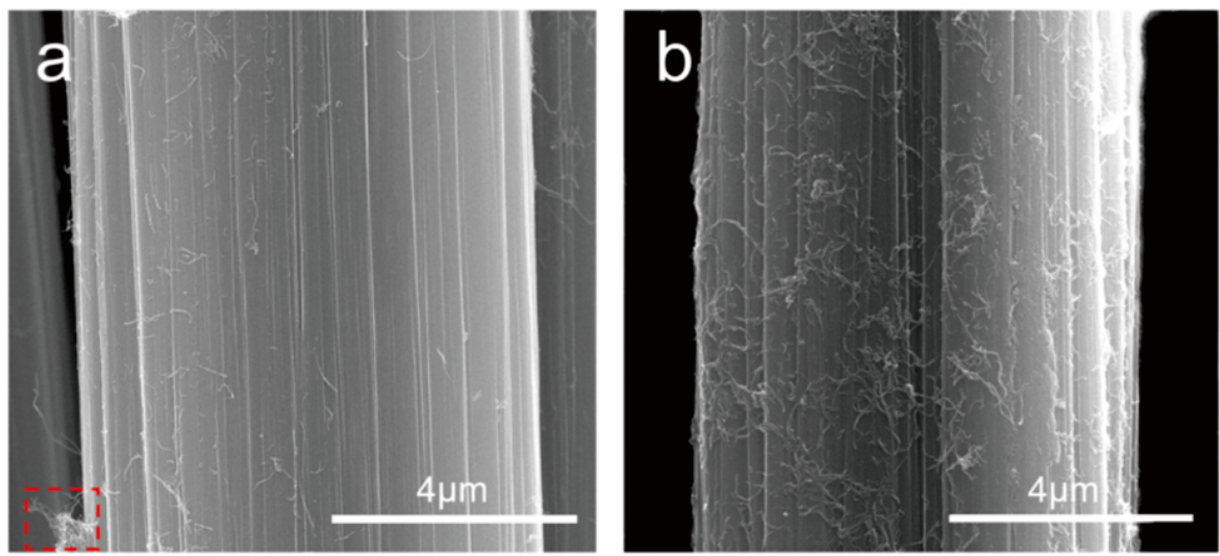

Fig. 9 SEM images of CF coated with a) R806-raw CNTs and b) R806-CNTs-Acryl. 
DCA tests were carried out to examine the effects of R806-CNTsAcryl on the surface energy and the results are summarized in Table.1. After anodic oxidation treatment, the contact angle of CFs in both polar and non-polar test mediums declined apparently. Therefore, the polar component and dispersion component of the surface energy all increased significantly, which is mainly attributed to the increased quantity of active functional groups. After grafted with acryloyl chloride, a large number of polar groups on fiber surface were consumed, thus the surface energy of CF-Vinyl decreased to 52.1 $\mathrm{mJ} / \mathrm{m}^{2}$.

The CA of CFs treated with R806-CNTs-Acryl in water and diiodomethane decreased to 58.9 and $18.3^{\circ}$, respectively. The contact angles in water for CFs treated with pure R806 sizing agent and R806CNTs-Acryl are nearly the same, which means that the introducing of CNTs-Acryl has almost no influence on the wettability of fiber surface and nearly all CNTs-Acryl are coated with R806 resin. The increased dispersion component was due to the increased roughness caused by CNTs-Acryl on the fiber surface and the increased polar component of the sizing agent treated CFs is attributed to the polar groups in R806 molecular chains.

Table. 2 is the surface elemental composition of carbon fibers before and after R806-CNTs-Acryl sizing agent treatment. As shown in Table 2, the $\mathrm{O} / \mathrm{C}$ ratio of raw $\mathrm{CF}$ is lowest among all samples. Owing to the introduction of oxygen-containing groups, the $\mathrm{O} / \mathrm{C}$ ratio of $\mathrm{CF}$ treated with anodic oxidation has risen to 0.282 . Due to the grafting reaction, some oxygen-containing groups have reacted with acryloyl chloride. Thus the $\mathrm{O} / \mathrm{C}$ ratio of CF-Vinyl has a slightly decrease, but still higher than that of raw CF. After treated with R806-CNTs-Acryl, the $\mathrm{N}$ element content of $\mathrm{CF}$ reduced obviously, which is due to the shielding effect of R806-CNTs-Acryl as an isolated layer. Moreover, the
$\mathrm{O} / \mathrm{C}$ ratio of $\mathrm{CF}$ treated with $\mathrm{R} 806-\mathrm{CNTs}-\mathrm{Acryl}$ is still higher than that of raw $\mathrm{CF}$.

Fig. 10 is the fitting curves of C1s spectra of CFs before and after R806-CNTs-Acryl treatment. After anodic oxidation treatment, the content of $\mathrm{C}-\mathrm{O}, \mathrm{C}=\mathrm{O}$ and $\mathrm{C}=\mathrm{O}-\mathrm{O}$ of fiber surface increased obviously, which is due to the introduction of a large number of polar groups. When compared to the surface groups of CFs treated with anodic oxidation, the $\mathrm{C}-\mathrm{O}$ content of CF-Vinyl decreased somewhat, but the $\mathrm{C}=\mathrm{O}-\mathrm{O}$ content increased, which is due to the esterification reaction between the hydroxyl groups on fiber surface and the acyl chloride groups of the acryloyl chloride. After treated with R806-CNTs-Acryl, the C-O content of CFs increased, which is due to the large number of hydroxyl groups in molecular structure of R806.

\subsection{The effect of R806-CNTs-Acryl on interfacial bonding of CF/UP composites}

The ILSS of CF/UP composites reinforced by different CFs were shown in Fig. 11. Compared to the raw CF composites, the ILSS of the CFVinyl composites increased by $8 \%$, which is due to the unsaturated double bonds reaction between the CF-Vinyl and the UP matrix.

For the composites reinforced by CFs which treated with R806CNTs-Acryl, the introduction of CNTs-Acryl obviously improved the interface bonding of the CF/UP composites. The ILSS increased from 47.7 MPa for the raw CFs to $61.23 \mathrm{MPa}$ for the CFs treated with R806CNTs-Acryl by $28.4 \%$. For one hand, the polar functional groups of sizing agent played an important role in improving the interfacial bonding between the CFs and the UP matrix. The polar groups could increase the wettability of CFs for UP matrix and strengthen the interactions of molecular in the interface region. For another hand, the rough surface caused by CNTs-Acryl contribute to the interlocking

Table 1 Contact angles and surface energy of carbon fibers.

\begin{tabular}{|c|c|c|c|c|c|}
\hline \multirow{2}{*}{ Carbon fiber } & \multicolumn{2}{|c|}{ Contact angle $\quad\left({ }^{\circ}\right)$} & \multicolumn{2}{|c|}{ Surface energy } & \multirow{2}{*}{$\frac{\left(\mathrm{mJ} / \mathrm{m}^{2}\right)}{\gamma}$} \\
\hline & Water & Diiodomethane & $\gamma^{\mathrm{d}}$ & $\gamma^{\mathrm{p}}$ & \\
\hline Raw CF & 71.3 & 27.3 & 45.3 & 5.4 & 47.7 \\
\hline Anodic oxidation & 55.8 & 24.1 & 46.7 & 12.3 & 59.0 \\
\hline CF-Vinyl & 69.2 & 25.8 & 45.9 & 6.2 & 52.1 \\
\hline R806 & 58.6 & 17.2 & 48.6 & 10.2 & 58.8 \\
\hline R806-CNTs - Acryl & 58.9 & 18.3 & 48.3 & 10.2 & 58.5 \\
\hline
\end{tabular}

Table 2 Surface elemental composition of carbon fibers.

\begin{tabular}{lllll}
\hline & \multicolumn{4}{c}{ Element content (\%) } \\
\cline { 2 - 5 } Carbon fiber & $\mathrm{C}$ & $\mathrm{N}$ & $\mathrm{O}$ & $\mathrm{O} / \mathrm{C}$ \\
\hline Raw CF & 82.42 & 3.67 & 13.91 & 0.169 \\
Anodic oxidation & 73.91 & 5.25 & 20.84 & 0.282 \\
CF-Vinyl & 76.45 & 3.34 & 20.21 & 0.264 \\
R806 -CNTs -Acryl & 78.06 & 1.82 & 20.12 & 0.258 \\
\hline
\end{tabular}


between fiber surface and matrix, which is beneficial to the improvement of interfacial bonding. For the CNTs-Acryl, R806 and MR13006, each of their molecular structure have double bonds, which could participate in the curing process of UP matrix through radical polymerization and form chemical bonding in different levels. Furthermore, the enhanced mechanical interlocking and the local mechanical properties of interface region are beneficial to transfer the load. Furthermore, the interface layer with gradient mechanical properties caused by CNTs-Acryl in sizing agent is not only beneficial to transfer and disperse stress, but also for eliminating the internal stress between CFs and UP matrix when curing is done.

Figs. $12 \mathrm{a} \& \mathrm{~b}$ shows the shear fracture surfaces of the composites treated with and without R806-CNTs-Acryl. As shown in Fig. 12a, plenty of raw fibers were pulled out from the UP resin and lots of voids appeared in the UP resin, indicating a weak interfacial bonding between CFs and the UP matrix.
After treated with R806-CNTs-Acryl, the interface crack reduced and a uniform layer of UP resin could be observed on the fiber surface. Furthermore, only a small number of fibers were pulled out and the UP and/or CF debris were found to be uniformly distributed throughout the cross section, all of which were attributed to the enhanced roughness and surface energy of the fibers, further confirming an enhanced interphase of the CFs and UP resin.

Fig. 13 shows the impact properties of CF/UP composites before and after interface modification. The initial and propagative energy of the raw CFs composites were 0.82 and $0.23 \mathrm{~J}$, respectively. The R806CNTs-Acryl treatment obviously increased the impact toughness of the $\mathrm{CF} / \mathrm{UP}$ composites. The initial and propagative energy of the composites treated with R806-CNTs-Acryl increased to 1.04 and $0.57 \mathrm{~J}$, respectively. According to traditional interface theory, strong interface bonding leads to stress concentration and goes against the movement of polymer molecular chains in the interface region, which result in the decrease of
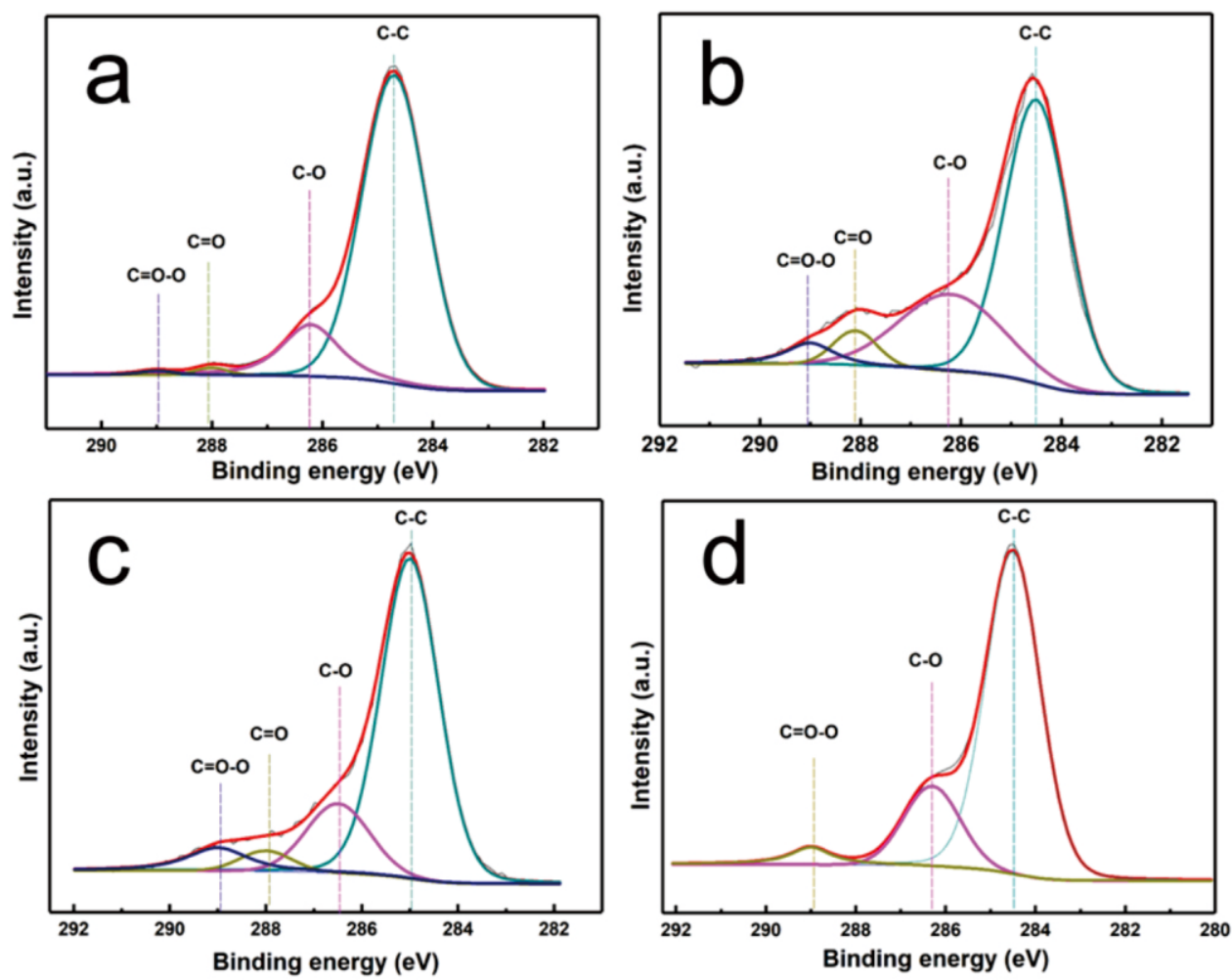

Fig. 10 Fitting curves of C1s spectra of CFs: a) Raw-CFs, b) Anodic oxidation, c) CF-Vinyl, d) R806-CNTs-Acryl.

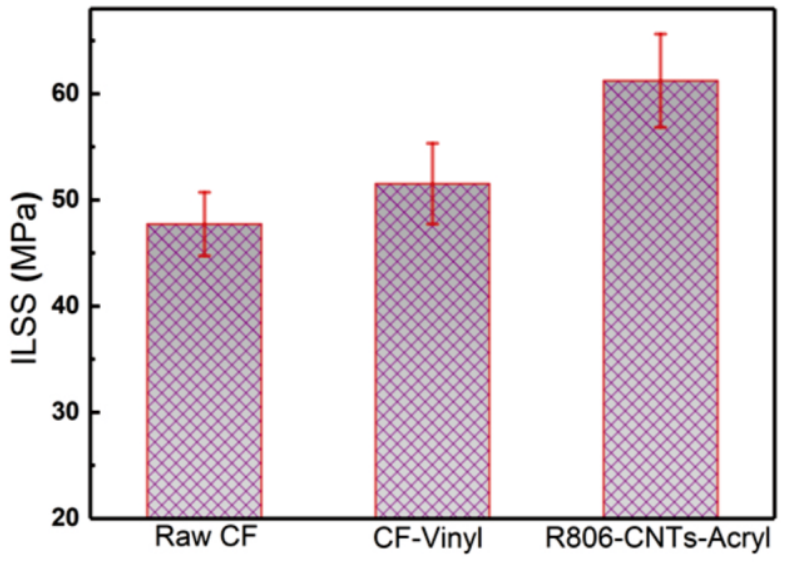

Fig. 11 ILSS of CF/UP composites reinforced by different carbon fibers. 
the impact property. ${ }^{38}$ However, surprisingly in our case, both ILSS and impact property were enhanced at the same time. The previous results show that CF-Vinyl is beneficial for increasing the ILSS of CF/UP composites. However, no significant improvement was observed for the impact properties of CF-Vinyl reinforced composites when compared to that of $\mathrm{CF} / \mathrm{UP}$ composites before modification, which illustrate the key role of CNTs for the improvement of the impact properties.

Introducing CNTs to vinyl ester sizing agent can structure the rough surface of $\mathrm{CFs}$, which restricts the movement of molecules in the interphase and improves the mechanical interlocking between CFs and UP resins. When the impact load was applied, the crack propagating orientation will be changed by CNTs and creates more tiny cracks in the bonding interface, which are helpful to dissipate the impact energy. Furthermore, the CNTs containing interphase with proper modulus between CFs and UP resin is also helpful to prevent the cracks from direct contacting the fiber surface and reduces the stress concentration in the interface region.

\section{Conclusions}

The R806 vinyl ester was the most effective for interfacial property enhancement after a screening test among R806, epoxy and MR13006. More active groups and better interfacial compatibility with UP make R806 a suitable polymer for preparing sizing agent for CF/UP composites when compared to commercial epoxy. R806-CNTs-Acryl was prepared to increase the interfacial properties of CF/UP composites. The amount of polar functional groups and the wettability of CFs were significantly enhanced after the coating treatment. The interlaminar shear strength (ILSS) and impact toughness were enhanced by 28.4 and $53.3 \%$, respectively. Introducing CNTs to vinyl ester sizing agent can structure the rough surface of $\mathrm{CFs}$, which restricts the movement of molecules in the interphase and improves the mechanical interlocking between CFs and UP resins. The R806-CNTs-Acryl effectively increased the interface compatibility of composites not only by using the reactive groups of R806 to improve the surface energy, but also reacting with UP matrix during the curing process. Furthermore, the CNTs containing interphase with proper modulus between CFs and UP resin is also helpful to prevent the cracks from direct contacting the fiber surface and reduces the stress concentration in the interface region.

\section{Acknowledgement}

Supported by Key Laboratory of Engineering Dielectrics and Its Application (HUST), Ministry of Education, Harbin innovation talent program of science and technology (2017RAQXJ105), University Nursing Program for Young Scholars with Creative Talents in Heilongjiang Province, Heilongjiang Natural Science Foundation (QC2017038).

\section{References}

1. D. Jiang, L. Liu, J. Long, L. Xing, Y. Huang, Z. Wu, X. Yan and Z. Guo, Compos. Sci. Technol., 2014, 100, 158-165.

2. D. Jiang, L. Xing, L. Liu, X. Yan, J. Guo, X. Zhang, Q. Zhang, Z. Wu, F. Zhao and Y. Huang, J. Mater. Chem. A, 2014, 2, 18293-18303.
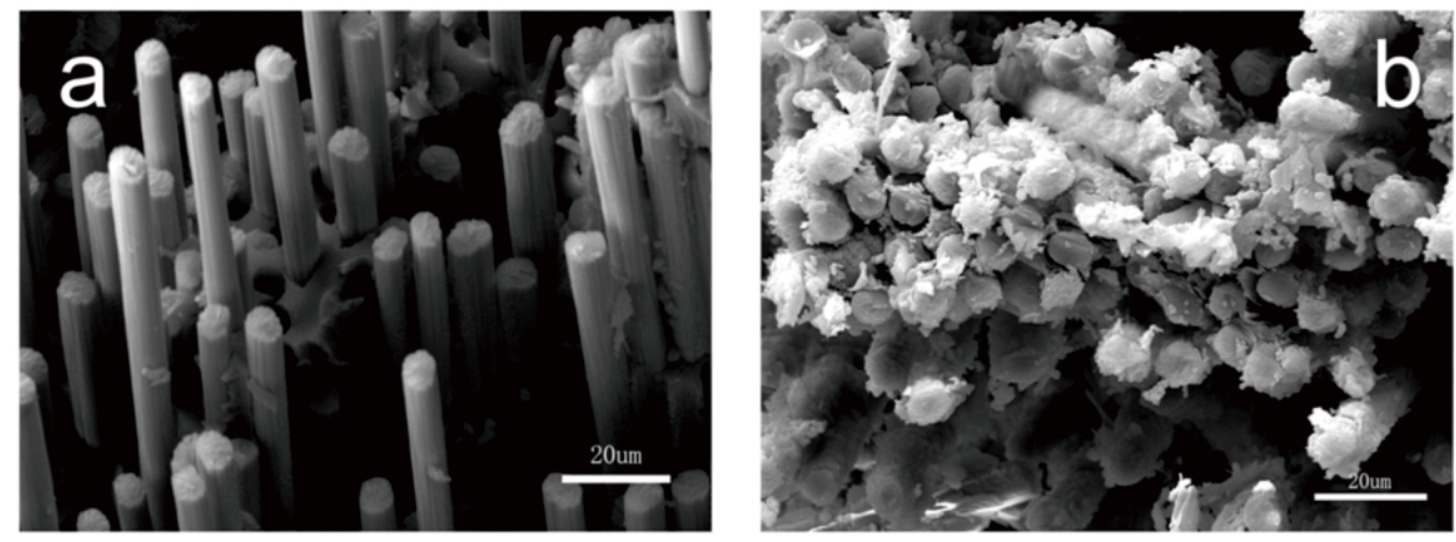

Fig. 12 Fracture images of composites reinforced with different fibers: a) Raw CF, b) R806-CNTs-Acryl.

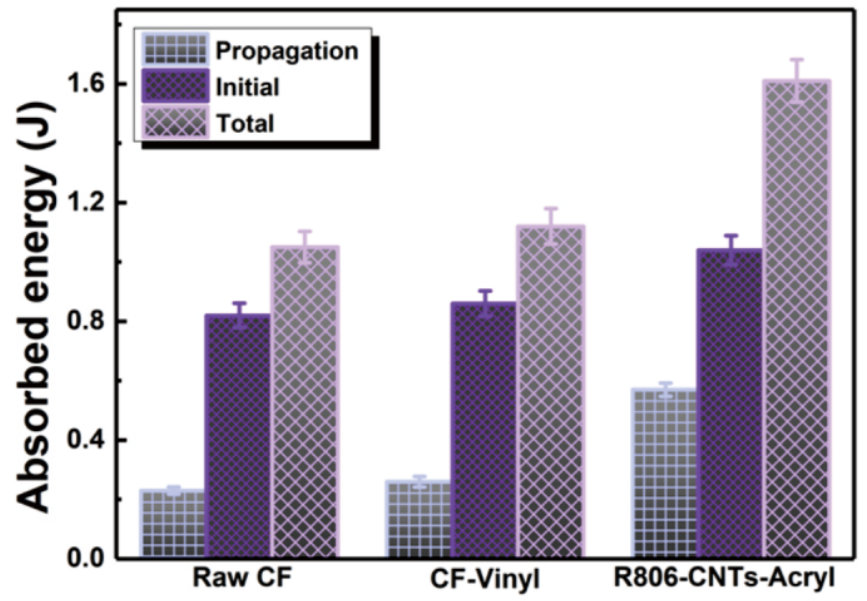

Fig. 13 Impact toughness testing results of CF/UP composites. 
3. D. Jiang, L. Xing, L. Liu, S. Sun, Q. Zhang, Z. Wu, X. Yan, J. Guo, Y. Huang and Z. Guo, Compos. Sci. Technol., 2015, 117, 168-175.

4. L. Ma, N. Li, G. Wu, G. Song, X. Li, P. Han, G. Wang and Y. Huang, Appl. Surf. Sci., S0169433217329598.

5. G. Wu, L. Chen and L. Liu, Compos. Part A-Appl. S., 98, 159-165.

6. Z. Hu, Q. Shao, M. G. Moloney, X. Xu, D. Zhang, J. Li, C. Zhang and Y. Huang, Macromolecules, 2017, 50.

7. Z. Hu, Q. Shao, Y. Huang, L. Yu, D. Zhang, X. Xu, J. Lin, H. Liu and Z. Guo, Nanotechnology, 2018.

8. K. Sun, P. Xie, Z. Wang, T. Su, Q. Shao, J. E. Ryu, X. Zhang, J. Guo, A. Shankar and J. Li, 2017.

9. M. Q. Tran, K. K. C. Ho, G. Kalinka, M. S. P. Shaffer and A. Bismarck, Compos. Sci. Technol., 2008, 68, 1766-1776.

10. G. Vuković, A. Marinković, M. Obradović, V. Radmilović, M. Čolić, R. Aleksić and P. S. Uskoković, Appl. Surf. Sci., 2009, 255, 8067-8075.

11. C. Wang, M. Zhao, J. Li, J. Yu, S. Sun, S. Ge, X. Guo, F. Xie, B. Jiang, E. Wujcik, Y. Huang, N. Wang and Z. Guo, Silver nanoparticles/graphene oxide decorated carbon fiber synergistic reinforcement in epoxy-based composites, 2017.

12. C. Wang, M. Zhao, J. Li, J. Yu, S. Sun, S. Ge, X. Guo, F. Xie, B. Jiang and E. K. Wujcik, 2017

13. Z. Wu, S. Gao, L. Chen, D. Jiang, Q. Shao, B. Zhang, Z. Zhai, C. Wang, M. Zhao and Y. Ma, Macromol. Chem. Phys., 2017, 218, 1700357.

14. C. Cheng, R. Fan, Z. Wang, Q. Shao, X. Guo, P. Xie, Y. Yin, Y. Zhang, L. An and Y. Lei, Carbon, 2017, 125.

15. H. Gu, H. Zhang, J. Lin, Q. Shao, D. P. Young, L. Sun, T. D. Shen and Z. Guo, Polymer, 2018.

16. Y. Guo, G. Xu, X. Yang, K. Ruan, T. Ma, Q. Zhang, J. Gu, Y. Wu, H. Liu and Z. Guo, J. Mater. Chem. C, 2018.

17. X. Fang, J. Tan, Y. Gao, Y. Lu and F. Xuan, Nanoscale, 2017, 9.

18. C. P. Feng, L. Bai, R. Y. Bao, Z. Y. Liu, M. B. Yang, J. Chen and W. Yang, Advanced Composites \& Hybrid Materials, 2018, 1, 160-167.

19. M. D. Hager, P. Greil, C. Leyens, d. Z. S. Van and U. S. Schubert, $A d v$. Mater, 2010, 22, 5424-5430.

20. J. Li, M. J. Vergne, E. D. Mowles, W. H. Zhong, D. M. Hercules and C. M. Lukehart, Carbon, 2005, 43, 2883-2893.
21. Y. He, S. Yang, H. Liu, Q. Shao, Q. Chen, C. Lu, Y. Jiang, C. Liu and Z. Guo, $J$ Colloid Interface Sci, 2018, 517, 40-51.

22. Z. Hu, Q. Shao, X. Xu, D. Zhang and Y. Huang, Composites Science \& Technology, 2017, 142, 294-301.

23. M. Zhao, L. Meng, L. Ma, L. Ma, X. Yang, Y. Huang, J. E. Ryu, A. Shankar, T. Li and C. Yan, Compos. Sci. Technol., 2018, 154

24. C. Wang, J. Li, J. Yu, S. Sun, X. Li, F. Xie, B. Jiang, G. Wu, F. Yu and Y. Huang, Compos. Part A-Appl. S., 101, 511-520.

25. X. Cui, G. Zhu, Y. Pan, Q. Shao, C. Zhao, M. Dong, Y. Zhang and Z. Guo, Polymer, 2018, 138.

26. H. Qian, E. S. Greenhalgh, M. S. P. Shaffer and A. Bismarck, J. Mater. Chem., 2010, 20, 4751-4762.

27. A. H. Barber, Q. Zhao, H. D. Wagner and C. A. Baillie, Compos. Sci. Technol., 2004, 64, 1915-1919.

28. C. Sellitti, S. Vargui, E. Martuscelli and D. Fabbro, J. Mater. Sci., 1987, 22 3477-3484

29. J. Chen, X. Cui, Y. Zhu, W. Jiang and K. Sui, Carbon, 2017, 114, 441-448.

30. M. Li, Y. Gu, Y. Liu, Y. Li and Z. Zhang, Carbon, 2013, 52, 109-121.

31. J. Huang, C. Mao, Y. Zhu, W. Jiang and X. Yang, Carbon, 2014, 73, $267-$ 274.

32. Y. Li, B. Zhou, G. Zheng, X. Liu, T. Li, C. Yan, C. Cheng, K. Dai, C. Liu and C. Shen, J. Mater. Chem. C, 2017.

33. K. Zhang, G. H. Li, L. M. Feng, N. Wang, J. Guo, K. Sun, K. X. Yu, J. B. Zeng, T. Li and Z. Guo, J. Mater. Chem. C, 2017, 5.

34. P. Zhang, X. Huang, J. Fu, Y. Huang and X. Tang, Macromol. Mater. Eng., 2010, 295, 437-441.

35. W. Zijian, M. Linghui, L. Li, J. Zaixing, X. Lixin, J. Dawei and H. Yudong, J. Reinf. Plast. Comp., 2013, 33, 242-251.

36. Q. Zhang, L. Liu, D. Jiang, X. Yan, Y. Huang and Z. Guo, J. Compos. Mater, 0021998314557298.

37. T. H. Cheng, J. Zhang, S. Yumitori, F. R. Jones and C. W. Anderson, Composites, 1994, 25, 661-670.

38. F. Zhao, Y. Huang, L. Liu, Y. Bai and L. Xu, Carbon, 2011, 49, 2624-2632.

Publisher's Note Engineered Science Publisher remains neutral with regard to jurisdictional claims in published maps and institutional affiliations. 